\title{
Oxidoreductase Gene
}

National Cancer Institute

\section{Source}

National Cancer Institute. Oxidoreductase Gene. NCI Thesaurus. Code C25939.

Oxidoreductase Genes encode the class of enzymes that catalyze oxidation/reduction reactions (Oxidoreductases). The oxidized substrate is regarded as a hydrogen donor. Systematic enzyme names employ dehydrogenase wherever possible, or reductase; oxidase is only used where $\mathrm{O} 2$ is the acceptor. 